\title{
La Vanité à l'usage des demoiselles : savoir-vivre et savoir-mourir dans le roman d'éducation pour filles dans le Second Empire
}

Et pourtant vous serez semblable à cette ordure, À cette horrible infection, Étoile de mes yeux, soleil de ma nature, Vous, mon ange et ma passion!

Oui ! telle vous serez, ô la reine des grâces, Après les derniers sacrements, Quand vous irez, sous l'herbe et les floraisons grasses, Moisir parmi les ossements.

Alors, ô ma beauté ! dites à la vermine Qui vous mangera de baisers, Que j'ai gardé la forme et l'essence divine De mes amours décomposés!

(Baudelaire, « Une charogne », 1861, p. 67)

La vanité révèle l'impermanence en faisant choir le masque de la beauté temporaire - ou temporelle - à dessein d'éperonner le désir de ce qui ne passe pas. Dans l'art religieux catholique, elle fait ainsi à la fois office de méditation et d'outil pédagogique. Cette ambivalence invite à s'interroger sur les modalités d'écriture de la vanité dans le champ de la littérature édifiante à l'usage des demoiselles. La vanité y oc-

Marie Kawthar Daouda - docteur ès lettres, Stipendiary Lecturer à l'Université d'Oxford - Oriel College. Adresse pour correspondance : Oriel College, Oxford, OX14EW, Royaume-Uni ; e-mail : marie. daouda@oriel.ox.ac.uk 
cupe une place centrale durant le Second Empire, singulièrement à la même époque où Baudelaire se faisait fustiger pour l'obscénité d' "Une charogne ", vanité d'autant plus sordide que l'animal s'y substitue à l'humain, la pourriture de la chair à la minéralité de l'os et le rire grinçant à la lamentation. Après la défaite de Napoléon, l'échec de la Deuxième République socialiste et démocrate, le triomphe de la bourgeoisie et des finances après le coup d'état de Napoléon III et l'exil de Victor Hugo, le cœur du XIX ${ }^{e}$ siècle français est un instant crucial où la perception de l'impermanence s'impose tant en art qu'en politique. Les vanités à l'usage des demoiselles sont-elles plus acceptables que l'art poétique de Baudelaire ? Comment les angoisses du temps filtrent-elles jusqu'aux individus les plus jeunes? Le Journal de Marguerite ou les deux années de préparation à la Première Communion (1858) et le cycle de Sophie ${ }^{1}$, personnage emblématique des romans de la Comtesse de Ségur, éclairent d'un jour singulier les enjeux de la Vanité durant ce temps de crise et de reconstruction qu'est la première décennie du Second Empire 2 . Le roman, emblématique des lectures édifiantes pour jeunes filles, a suffisamment marqué son temps pour qu'Edmond de Goncourt en fasse en 1884 l'une des lectures de Chérie, jeune fille condamnée à une mort précoce par une hérédité corrompue.

Le roman d'éducation à l'usage des demoiselles a une fonction particulière. S'il s'inscrit dans un programme deéducation, visant à incorporer le personnage et, à travers elle, la lectrice, au monde des adultes, cette dimension éducative est indissociable d'un programme religieux. C'est par sa foi en les principes qui lui auront été inculqués que la jeune fille parviendra à assumer son rôle familial et social. La confrontation à la mort relève donc, sinon de l'évidence, du moins de la nécessité. La vanité peut-elle être exploitée comme ars moriendi à destination d'un lectorat dont la vie sociale n'a pas encore commencé ? Y a-t-il un lien entre les angoisses d'un Baudelaire ou d'un Goncourt face à la labilité et l'enseignement délivré à leurs contemporaines via la présence de la vanité dans le roman d'éducation?

Le parti-pris catholique de Victorine Monniot et de la Comtesse de Ségur sollicite la vanité dans deux perspectives. La première revient à enseigner le détachement du monde. Elle a pour but de corriger les défauts d'enfant derrière lesquels se profilent les péchés capitaux des adultes. La seconde, plus complexe, relève du rapport à la mort du corps incarné. Il s'agira de voir comment ces deux pôles s'articulent et se complètent dans la construction d'un devoir religieux - souvenir des morts et souvenir à rebours de l'éternité à venir - dont l'enjeu, en somme, n'est pas si distant de la clausule qui fait de la dernière strophe d' "Une charogne » un poignant dialogue entre ce qui n'est plus et ce qui se sait fini.

1. Les Malheurs de Sophie (1858), Les Petites filles modèles (1859), Les Vacances (1859).

2. Baudelaire écrivait : «De Maistre et Edgar Poe m’ont appris à raisonner» (2004, p. 401). Le théoricien du sacrifice était, durant son séjour à Saint-Pétersbourg, suffisamment proche de la famille Rostoptchine pour avoir joué un rôle décisif dans la conversion de la mère de la Comtesse de Ségur. Victorine Monniot, fervente catholique légitimiste, avait sans doute les mêmes références culturelles. 


\section{Châtiment de l'orgueil}

Le lectorat-cible de Victorine Monniot dans Le Journal de Marguerite et celui de la Comtesse de Ségur dans le cycle de Sophie est sensiblement le même et repose sur une identification entre les jeunes lectrices et les héroïnes qui se préparent à la première communion. Ce terme est indiqué dès le sous-titre par Victorine Monniot et figure implicitement chez la Comtesse de Ségur avant d'être révélé lorsque s'achève Les Vacances. Philippe Lejeune souligne le caractère spécifiquement féminin et religieux du journal intime comme exercice de préparation spirituelle ${ }^{3}$. Le journal intime fictif que propose Victorine Monniot est initiatique. Il propose un chemin édifiant d'initiation chrétienne qui passe par l'intériorisation des bienfaits de la mortification comme châtiment de l'orgueil. Dans Le Journal de Marguerite, la première faute grave que rapporte la fillette dans son journal, qu'elle écrit comme une confession, est une colère violente face à laquelle sa gouvernante, Caroline Valmy, demande :

Était-ce un être raisonnable que j’a contemplé, hier, dans cette petite fille à l'oeil étincelant, aux poings levés, aux attitudes menaçantes, à l'air insensé enfin, dont le triste souvenir m'a poursuivie toute la nuit ? [...] Je pleure sur vous, lorsque je me demande quelle sera votre vie si vous ne domptez pas la violence de votre caractère. (Monniot, 1886, p. 21)

Les colères de Marguerite au début de son parcours sont explicitement liées à son orgueil, que ce soit à l'encontre de son frère et de ses sœurs ou à l'égard de sa gouvernante. L'éducation du jeune enfant a pour but premier de brider sa sensualité et son impétuosité, c'est-à-dire de l'extraire de la temporalité immédiate de l'enfance pour l'intégrer à celle du monde des adultes, qui s'inscrit dans la durée et tend, dans un référentiel catholique, vers la vie éternelle. La récompense du sens de la mortification, pour différée qu'elle soit, est présentée comme indéniable.

Dans Les Malheurs de Sophie, la fillette se retrouve en rêve face à deux chemins, l'un agréable, sablé, bordé de fleurs, l'autre chargé de cailloux et de ronces. L’ange gardien de la fillette lui annonce de grands malheurs si elle emprunte le chemin qui a l'air si plaisant, et face à l'entêtement de Sophie, il lui promet qu'il l'attendra éternellement au bout du chemin apparemment ardu, mais qui mêne à la félicité. Cette allégorie est clairement expliquée par l'auteur elle-même, relayée par la mère de Sophie :

3. «Il est dramatiquement organisé autour de la première communion, qui est la grande épreuve d'initiation à la fois spirituelle et sociale pour les petites filles de cette classe. On commence son journal à dix ans, on attend, on se prépare... [...] Aucun journal de garçon, aucun roman qui fasse le pendant du Journal de Marguerite, alors qu'après tout on ne voit pas pourquoi les petits garçons n'auraient pas eu à améliorer leur vertu et à former leur style. Ce type de journal est vraiment "mère-fille". Le garçon, lui, apprend le latin, des choses qui ne sont pas pour les filles, et ses relations avec son précepteur ne sont pas du même ordre » (Lejeune, 1993, p. 20-21). 
C'est que le bon Dieu, qui voit que tu n'es pas sage, te prévient par le moyen de ce rêve que, si tu continues à faire tout ce qui est mal et qui te semble agréable, tu auras des chagrins au lieu d'avoir des plaisirs. Ce jardin trompeur, c'est l'enfer ; le jardin du bien, c'est le paradis ; on y arrive par un chemin raboteux, c'est-à-dire en se privant de choses agréables, mais qui sont défendues; le chemin devient plus doux à mesure qu'on marche, c'est-à-dire qu'à force d'être obéissant, doux, bon, on s'y habitue tellement que cela ne coûte plus d'obéir et d'être bon, et qu'on ne souffre plus de ne pas se laisser aller à toutes ses volontés. (Ségur, 1929, p. 154)

Le parcours de la fillette est donc présenté, en allégorie, par ces deux chemins la voie des plaisirs et celle, plus ardue, des mortifications et des corrections. Les chapitres où elle a lieu s'ouvrent de manière programmatique par "Sophie était... » associé au défaut à corriger : "Sophie était étourdie » (Ségur, 1929, p. 27), puis à deux reprises « Sophie était gourmande » (1929, p. 69 et 79), "Sophie était coquette » (1929, p. 53) et surtout, "Sophie était colère ». Parmi les petits péchés capitaux de l'enfance, l'orgueil et la colère, qui en découle (Dupanloup, 1869, p. 208 sq.), sont les cibles privilégiés des éducateurs. Les ouvrages édifiants transmettent un enseignement sur la tempérance comme discipline intérieure, visant à dompter la sensualité enfantine si vivement décriée par Monseigneur Dupanloup, contemporain de la comtesse de Ségur. Dans le sillage de la scolastique, la littérature édifiante et les ouvrages d'éducation s'accordent à esquisser un parallèle entre l'intempérance du corps et l'orgueil de l'esprit. Monseigneur Dupanloup écrit :

L'orgueil, la vanité, l'amour-propre, comme immodestie, consiste à ne pas se contenir, à ne pas se respecter, à se flatter, à s'idolâtrer misérablement soi-même. L'orgueil est donc l'ostentation, l'immodestie, l'impudence, l'incontinence de l'esprit, comme l'impureté est l'impudence, l'immodestie, l'incontinence et en quelque sorte l'orgueil du corps. (1869, p. 23)

Est enfant tout ce qui ne sait pas saisir la vanité des plaisirs et des passions. Comme l'écrit Laura Kreyder, « ce qui atterre l'éducateur chrétien, c'est donc la sensualité primordiale qui s'exprime chez l'enfant, le désir de la jouissance, l'attachement au Moi » (1987, p. 143). Dès lors, toute l'éducation, la sortie hors de l'enfance, tendra vers le châtiment de l'orgueil. Par l'intermédiaire de Caroline Valmy s'adressant à ses petites élèves, Victorine Monniot explique à ses lectrices :

[L]'orgueil se manifestera par la vanité, qui est un amour immodéré de plaire et qui se nourrit de puériles louanges, de frivoles pensées. Ce qu'il y a de plus périssable au monde - les avantages extérieurs - leur deviendra précieux. Ils garderont dans leur esprit certains compliments flatteurs, qui leur auront été donnés par affection, par indulgence ou par politesse, et trouveront une douceur dangereuse à repasser ces agréables souvenirs, qui finiront bientôt par détourner leur âme des réflexions utiles et des saintes pensées. (1886, p. 286) 
L'objectif de cet enseignement est d'inculquer la différence entre " ce qu'il y a de plus périssable au monde » et les "saintes pensées ». Comme chez les moralistes du XVII siècle dont Victorine Monniot se fait l'écho, l'enseignement sur les vanités dévoile l'attrait trompeur des choses périssables.

Si la gourmandise de Sophie est payée par une mémorable indigestion, les contre-exemples offerts aux jeunes lectrices sont tout aussi édifiants. La camarade d'enfance de Marguerite, Clara de Baldi, jouit d'une ample fortune mais est mal élevée ; au terme de Marguerite à vingt ans, suite et fin du journal de Marguerite, elle est en proie au désenchantement ennuyé et aigri des heureux du monde. Chez la Comtesse de Ségur, la vanité des biens matériels est enseignée à travers le ridicule de mademoiselle Yolande Tourne-Boule, fillette de la bourgeoisie brusquement enrichie, qui s'enorgueillit de biens dont elle ne dispose à aucun titre :

Papa me donne tout ce que je veux [...]. J'ai déjà des parures de diamants, de perles et de rubis. La toilette que vous me voyez n'est rien auprès de celles que j'ai à Paris ; j'ai plus de cinquante robes et coiffures de bal, autant de robes de dîners et de visites. Maman a tous les jours une robe neuve; elle dépense cinquante mille francs par an pour sa toilette. (Ségur, 1884, p. 291-292)

Quand le petit Léon l'appelle "ma pauvre demoiselle ", Yolande éclate de rire, ce qui fait répondre à Camille, exemple de l'éducation réussie :

Ne riez pas, ma pauvre demoiselle ; ne riez pas! Vous êtes en effet à plaindre, Léon a raison : vous êtes pauvre de bonté, pauvre de charité, pauvre d'humilité, pauvre de raison et de sagesse. Vous voyez bien que vous n'avez pas la vraie richesse, et que si vous perdiez votre fortune, il ne vous resterait plus rien. (1884 p. 293)

C’est à Paul, sanctifié par l'expérience ascétique du naufrage et de la vie sur une île peuplée de sauvages, que revient d'exprimer la maxime de l'épisode :

C'est vrai. Quand on a de quoi manger, de quoi s'habiller, se chauffer et vivre agréablement, de quoi donner à tous les pauvres des environs, à quoi sert le reste ? On ne peut pas dîner plus d'une fois, monter sur plus d'un cheval, dans plus d'une voiture, brûler plus de bois que n'en peuvent tenir les cheminées. Ainsi, que faire du reste, sinon le donner à ceux qui n'en ont pas assez ? (1884, p. 202-203)

Tout au long du Journal de Marguerite, Victorine Monniot délivre le même enseignement de tempérance opposée à la vanité de la surabondance en ce monde. Cet enseignement voilé est exprimé plus clairement cinq ans plus tard lorsque Victorine Monniot offre à ses lecteurs la suite du parcours de Marguerite. À un public plus âgé, elle n'hésite pas à révéler le fond biblique sur lequel reposait l'éducation chrétienne de Marguerite enfant. Lorsque Marguerite visite le Mémorial de Sainte-Hélène, l'épo- 
pée napoléonienne et le spectre de l'empereur déchu mêlent un écho de l'Ecclésiaste à la fin de Nabuchodonosor : "Vanité des vanités! Tout n'est que vanité, hormis d'aimer Dieu et de le servir. Ces paroles semblent écrites en traits de flammes sur le sol de Sainte-Hélène » (Monniot, 1863, p. 64, V. Monniot souligne.) Plus loin, la fillette devenue jeune fille, dépossédée de toute sa fortune après le décès de son père, se réfère aux Béatitudes et à la vie des cénobites ou des franciscains :

Ne dirait-on pas que la Providence a voulu nous prouver, par un enseignement visible, que les richesses matérielles attachent à la terre, et que le malheur rapproche du Ciel ? [...] La misère ne saurait être affreuse, lorsque l'on s'y résigne. Est-ce que les saints ne l'ont pas supportée, aimée, appelée ? Leur paraissait-elle trop rude, à ces mendiants héroïques qui, dépouillés de toutes choses pour l'amour de Jésus-Christ, suivaient leur maître pauvre, dans un dénûment complet? (Monniot, 1863, p. 182-183)

Des premières bévues des petites filles modèles à la sagesse de Camille et de Paul, des colères orgueilleuses de Marguerite à sa maturité à vingt ans, le parcours d'éducation de la demoiselle vise à lui mettre sous les yeux la vanité des biens de ce monde et la nécessité de se faire, selon la parabole évangélique, un trésor de vertus impérissables.

\section{Présence de la mort}

Le lecteur contemporain pourrait s'étonner davantage de la présence de la mort sous la plume de Victorine Monniot que des traitements violents infligés aux enfants chez la Comtesse de Ségur. Cette dernière écrit, avec un certain détachement : "Les vacances étant finies, nous laisserons grandir, vivre et mourir nos amis sans plus en parler " (1859, p. 341). L'expérience de lecture, avec son commencement et son terme, est déjà en elle-même une initiation à la fin : le récit s'achève ; les personnages, une fois éduqués, grandissent, voient leur sort réglé en un bref paragraphe qui insiste sur le succès de cette éducation édifiante et sur la déchéance de mademoiselle Yolande devenue comédienne; puis meurent. Loin d'être propre à un Second Empire qui nous est déjà lointain, la présence de la mort dans ces romans pour enfants était loin de faire l'unanimité ; en témoigne le besoin qu'a Victorine Monniot de se justifier, lorsqu'elle publie la suite du Journal de Marguerite :

À ceux qui trouvent que les aventures de Marguerite sont « trop tristes » et qu'il ne faut pas parler de malheur aux enfants :

ils souffriront bien davantage, car ils ne sauront pas souffrir. Et ne dites point qu'il n'est pas besoin d'apprentissage vis-à-vis de la douleur ; vous prouveriez une trop grande ignorance des choses chrétiennes. (Monniot, 1863, p. 8) 
Dans la perspective de Victorine Monniot, de manière plus flagrante encore que chez la Comtesse de Ségur, il s'agit moins d'éduquer les enfants que de leur transmettre une éducation proprement chrétienne, où l'ars vivendi tend nécessairement vers un ars moriendi. Les prémisses de cet enseignement sont l'immortalité de l'âme et son corollaire, la singularité de l'individu face au jugement divin. Comme le souligne avec justesse Anne Bergier, l'éducation de Sophie repose d'abord sur sa séparation d'avec l'animal, grâce à laquelle l'enfant est appelée à prendre conscience du « «pacte anthropothéocratique » (Bergier, 2012, p. 432). Sophie découvre la décomposition de la chair lorsque, ayant plongé une tortue de terre dans l'eau pour la rafraîchir, elle s'aperçoit avec son cousin Paul que la bête est morte : "Enfin, un jour, en la mettant sur l'herbe, ils s'aperçurent qu'elle sentait mauvais. "Elle est morte, dit Paul; elle sent déjà mauvais." [...] Ainsi finit la pauvre tortue, qui fut le dernier animal qu'eut Sophie » (Ségur, 1858, p. 243-246). La phrase " elle sent déjà mauvais », sous la plume de la comtesse de Ségur, fait nécessairement écho au "Jam foetat » (Jn 11: 39), « il sent déjà mauvais », qui précède la résurrection de Lazare. C'est là que s’établit pour la comtesse la frontière entre animal et humain : la condamnation de l'animal à la décomposition, et la vocation du spirituel à la Résurrection.

La mort de la tortue clôt un enseignement commencé dès le début du roman, lorsque les enfants organisent les funérailles d'une poupée :

Quand la procession arriva au petit jardin de Sophie, on posa par terre le brancard avec la boîte qui contenait les restes de la malheureuse poupée. Les enfants se mirent à creuser la fosse ; ils y descendirent la boîte, jetèrent dessus des fleurs et des feuilles, puis la terre qu'ils avaient retirée ; ils ratissèrent promptement tout autour et y plantèrent deux lilas. Pour terminer la fête, ils coururent au bassin du potager et y remplirent leurs petits arrosoirs pour arroser les lilas. [...] On n'avait jamais vu un enterrement plus gai. Il est vrai que la morte était une vieille poupée, sans couleur, sans cheveux, sans jambes et sans tête, et que personne ne l'aimait ni ne la regrettait. (Ségur, 1929, p. 15)

Ce passage souligne d'abord le décalage entre l'humain et le simulacre. La procession est un entraînement parodique. Pour autant que ces gestes imitent les funérailles humaines, ils montrent également le peu d'importance de l'enveloppe vide et décomposée, du corps qui n'a pas plus d'âme que de couleur, de cheveux, de jambes, de tête, et qui est, de surcroît, dépouillé d’affections humaines. L'enterrement de la poupée et la mort de la tortue soulignent, par exclusion, que l'espérance est réservée à l'humain. Ces deux fausses morts annoncent la vraie épreuve de Sophie, confrontée au décès de sa mère entre Les Malheurs de Sophie et Les Petites filles modèles puis confiée à madame Fichini, sa belle-mère, dont la mort clôt Les Vacances et le parcours d'initiation au terme duquel Sophie est capable de se tenir de manière exemplaire auprès du lit d'agonie de sa marâtre, lui pardonnant ses mauvais traitements et promettant de prendre soin de la fille de celle qui fut sa tortionnaire (Ségur, 1884, p. 319-334). Les récits de la Comtesse de Ségur peuvent sembler plus légers, plus 
joyeux que Le Journal de Marguerite. Pourtant, l'enseignement délivré puise au même creuset. Monsieur de Rosbourg transmet aux enfants un enseignement sur la vanité de la vie :

Mes pauvres enfants, c'est toujours ainsi dans le monde ; le bon Dieu nous envoie des peines, des chagrins, des souffrances, pour nous empêcher de trop aimer la vie, et pour nous habituer à la pensée de la quitter. Quand tu seras plus grande, ma petite Marguerite, tu comprendras ce que Paul comprend très-bien déjà : c'est que, pour bien et chrétiennement mourir, il faut bien et chrétiennement vivre, souffrir ce que le bon Dieu nous envoie, être charitable pour tout le monde, aimer Dieu comme notre père, les hommes comme nos frères. (Ségur, 1884, p. 341)

L'éducation chrétienne telle que l'entreprennent la Comtesse de Ségur et Victorine Monniot apprend aux enfants au seuil de leur existence sociale comment bien quitter l'existence terrestre. Dès lors, la vie est tout entière orientée vers la «bonne mort». L'héroïne se dissocie d'un corps dont elle a appris qu'il était condamné à mourir, d'avantages temporels dont elle sait désormais qu'ils sont temporaires, et intègre une temporalité orientée vers l'éternité, où la mort n'est pas un terme mais l'ultime épreuve.

\section{La bonne mort et « l'essence divine "}

Les scènes de mort, que ce soit chez la Comtesse de Ségur ou chez Victorine Monniot, permettent de composer avec ce que Vladimir Jankélévitch désigne comme la « primultimité » (Jankélévitch, 1977, p. 300-320) de la mort, son caractère non pas universel mais au contraire éminemment singulier, donc tout aussi impossible à préparer qu'à éviter. Madame Fichini, la belle-mère cruelle et violente de Sophie, meurt trahie par un ancien bagnard pour qui elle a abandonné Sophie en croyant épouser un comte. Au lieu du paisible assoupissement de la « bonne mort », la vaniteuse séduite par les vanités du monde connaît une profonde angoisse. Sophie, éduquée au pardon par l'enseignement reçu à Fleurville, peut lui offrir un mot de pardon consolateur qui fait d'elle l'égale de Monsieur de Rosbourg et du curé dans les remerciements de la mourante :

« Merci, Sophie, merci... Grâce à toi... grâce à ton tuteur... et à ce bon curé... je meurs plus tranquille... Priez tous pour moi... Que Dieu me pardonne... Adieu, Sophie... ton père... pardonne... Je souffre... J'étouffe... Ah!"

Une convulsion lui coupa la parole. M. de Rosbourg saisit Sophie, terrifiée, dans ses bras, l'emporta dans la chambre voisine, la remit entre les mains de Hedwige et revint se mettre à genoux près du lit de Fichini, qui ne tarda pas à rendre le dernier soupir. (Ségur, 1884, p. 336-337) 
La mort n'est pas effrayante en elle-même pour qui, ayant assimilé l'enseignement sur les vanités, s'y est préparé toute sa vie durant de sorte à éviter remords et angoisses. Aussi, monsieur de Rosbourg déclare aux enfants : "Nous mourrons tous un jour; prions Dieu de nous faire vivre en chrétiens, pour que nous ayons une mort douce, pleine d'espérance et de consolation » (Ségur, 1884, p. 332) et poursuit à propos de madame Fichini : « Le bon Dieu aura pitié d’elle, car elle parait être bien sincèrement repentante » $(1884$, p. 332) dispensant ainsi un enseignement sur le repentir et la persévérance finale.

Ces ouvrages transmettent ainsi un art de « voir mourir » par lequel le spectacle de la mort n'est plus qu'un entraînement permettant de contourner, grâce au roman, la primultimité de la mort, en offrant à la jeune lectrice de faire par procuration l'expérience de ce qui n'est vécu qu'une seule fois. Diamétralement opposées à la mort inquiète de madame Fichini, les morts auxquelles est confrontée Marguerite sont en tout point édifiantes. Sa meilleure amie Marie, jeune orpheline aussi pieuse que douce, meurt quelques jours après la première communion de Marguerite. De manière profondément pédagogique, la Communion est à la fois le terme du parcours d'initiation et une étape vers le but ultime du sacrement, la pleine adhésion à la volonté de Dieu par l'incorporation au Christ. Marie mourante est plus que jamais l'exemple de "vertu héroïque » dont Marguerite fait son modèle édifiant : "Jamais [le prêtre] n'avait vu nulle part tant de foi, de résignation, de courage et d'amour, que dans cette jeune fille qui voyait venir la mort avec un si grand calme et une joie si profonde. [...] Les hommes mêmes [...] étaient dans l'étonnement et disaient "c'est un sublime spectacle" " (Monniot, 1886, p. 579). Aussi admiranda qu'imitan$d a$, Marie, qui a assimilé l'enseignement que Marguerite est en train de recevoir, échappe à l'angoisse - division de l'âme - autant qu'à la corruption de la chair - division du corps : "On aurait dit une vierge de marbre, et sa beauté était si frappante, que tout le monde était dans l'admiration autant que dans les larmes » (1886, p. 388). À l'inverse de la charogne de Baudelaire et à l'instar de l'ange de "Réversibilité " (Baudelaire, 1861, p. 100) - un autre nom pour le dogme de la Communion des Saints si cher à Victorine Monniot - le cadavre de Marie conserve sa «forme et son essence divine " maintenues dans le souvenir de Marguerite, qui n’hésite pas à la canoniser, faisant de son amie morte son ange protecteur et déclarant à Caroline Valmy : "Ne croyez-vous pas que cet ange exerce sur ma destinée quelque mystérieuse influence ? Elle m'attire dans une voie plus sainte, elle m'appelle à me rapprocher de Dieu...» (Monniot, 1863, p. 245). L'influence de Marie est en effet décisive. Si l'enseignement de la Comtesse de Ségur montre le vice puni et la vertu récompensée, celui de Victorine Monniot est sans doute davantage orienté vers l'éternité, glorifiant plutôt la longanimité des bons que leur succès matériel. Chez Monniot, la vanité sert moins à enseigner que la course aux plaisirs fait perdre le bonheur terrestre qu'à montrer combien le détachement des biens de ce monde entrouvre les portes du Ciel. Au terme de Marguerite à vingt ans, Marguerite meurt au monde en se faisant religieuse. 
Pour difficile qu'il soit dévaluer le succès de l'imprégnation par la lecture, annoncé comme projet dès la préface du Journal de Marguerite, ce roman en particulier a suscité l'enthousiasme de tout une génération conquise à ce désir d'édification. Edmond de Goncourt peut montrer la jeune Chérie gagnée par l'enthousiasme de renonciation et de mortification des petites hérö̈nes de Victorine Monniot :

En lisant ce livre d'une élégante réalité, Chérie éprouvait un sentiment, un sentiment nouveau que ne lui avait procuré jusqu'alors la lecture d'aucun livre. Il se faisait en elle, dans une espèce d'exaltation bizarre, la substitution de son moi dans toutes les choses exécutées ou dites ou pensées par la petite voyageuse, et elle prenait une part un peu fiévreuse à cette gentillesse de conduite, à ces bonnes intentions des actes, à ces élancements de religiosité, à ces attendrissements fervents, à cette aimable sanctification de Marguerite. Cette fusion de son être avec l'héroïne du livre la gratifiait d'une jouissance infinie, d'une absence d'elle-même ineffable, dans laquelle elle ne savait trop ni ce que qui se passait autour d'elle, ni ce qu'on lui disait, ni même ce qu'elle répondait. (Goncourt, 1884, p. 76)

Faut-il voir en Chérie une petite fille modèle manquée, une Marguerite du mal fanée faute d'avoir pu trouver sa place dans la décadence de l'Empire, faute d'avoir pu échapper à la foire aux vanités dont Edmond de Goncourt a vu l'effondrement à Sedan ? À la mort de marbre de Marie, Chérie oppose une décompostition dans des nuances cadavériques. Dépourvue de "forme et d'essence divine », son cadavre n'est même pas décrit. Dans l'avant-dernier chapitre du roman, juste avant le fairepart de deuil qui le clôt, elle est déjà morte : «[E]lle l'entendait [l’opéra] à travers une succession de demi-évanouissements, où le rouge qu'elle s'était fait mettre par Lina devenait par moments, sur la lividité de son teint, une tache brune, une tache effrayante " (Goncourt, 1884, p. 355-354). Chérie n'est-elle qu'une mauvaise lectrice, ou incarne-t-elle au contraire, en sa qualité d'allégorie du Second Empire, le point aveugle de l'enseignement sur la bonne mort, qui concerne non pas la mort des êtres mais la fin des empires ? Trente ans après Le Journal de Marguerite, Chérie dévoile l'insignifiance, la vanité des vanités.

La présence de la vanité dans le cycle de Sophie et dans Le Journal de Marguerite a une indéniable portée pédagogique. Elle propose à l'enfant un modèle de tempérance, par lequel la petite fille est appelée à renoncer autant à l'orgueil du corps qu'à celui de l'esprit. La présence de la mort dans ces romans a autant pour but de détacher la jeune fille des biens matériels que de lui enseigner un ars moriendi cautionnant son « essence divine ». Dépositaire d'une âme immortelle, elle peut dès lors renoncer à tout ce qui ne contribue pas à sa sanctification et assure, par sa disponibilité sacrificielle, la permanence d'un monde héroïque fondé sur les valeurs du sacrifice. La vanité lui enseigne à se former sur le modèle de la sainte ou de l'ange intercesseur. Après l'effondrement du Second Empire, il ne reste de cet idéal que la hantise fin-de-siècle de la décomposition et son corollaire, l'obsession symboliste pour les « apparitions 
disparaissantes ", les spectres immatériels et bienveillants dont la procession enseigne aussi bien l'impermanence de toute chose que l'éternité de l'immatériel.

\section{RÉFÉRENCES}

Baudelaire, Ch. (1861). Les Fleurs du mal. Paris, France : Poulet-Malassis.

Bergier, A. E. (2012). Quand Sophie aimait les bêtes. Autobiographie et animalité chez la Comtesse de Ségur. Dans Mariette-Clot C. et Zanone D. La Tradition des romans de femmes, XVIII'XIX' siècles (p. 413-438). Paris, France : Champion.

Bergier, A. E. (2004). CEuvres complètes. Paris, France : Robert Laffont.

Dupanloup, F. (1869). L'Enfant. Paris, France : Douniol.

Goncourt, E. de. (1884). Chérie. Paris, France : Charpentier.

Jankélévitch, V. (1977). La Mort. Paris, France : Flammarion.

Kreyder, L. (1987). L'Enfance des saints et des autres - Essai sur la Comtesse de Ségur. Bari, Italie : Schena-Nizet.

Lassère, M. (1999). Victorine Monniot, ou léducation des jeunes filles au XIX ${ }^{e}$ siècle. Paris, France : L'Harmattan.

Lejeune, Ph. (1993). Le Moi des Demoiselles, Enquête sur le journal de jeune fille. Paris, France : Seuil.

Monniot, V. (1886). Le Journal de Marguerite [1858]. Paris, France : Périsse frères.

Monniot, V. (1863). Marguerite à vingt ans. Paris, France : Périsse frères.

Reverzy, E. (2015). Raconter l'Empire : Chérie. Cahiers Goncourt, 2015, 73-84.

Ségur, S. de. (1929). Les Malheurs de Sophie [1858]. Paris, France : Hachette.

Ségur, S. de. (1859). Les petites filles modèles. Paris, France: Hachette.

Ségur, S. de. (1884). Les Vacances [1859]. Paris, France : Hachette.

RÉSUMÉ : Les années 1850 voient s'installer le Second Empire après un demi-siècle houleux où s'illustre la vanité du pouvoir temporel. Toutes deux légitimistes, Sophie Rostoptchine, comtesse de Ségur, et Victorine Monniot, ont laissé une empreinte profonde dans l'éducation des jeunes filles du Second Empire. Leur enseignement, fondé sur une interprétation concrète du catéchisme catholique, illustre les principes chrétiens de renonciation aux biens matériels enseignés par le memento mori tout en se faisant l'écho des angoisses de l'époque. L'article se propose d'étudier la présence de la vanité comme outil pédagogique dans Le Journal de Marguerite (1858) et le cycle de Sophie (1858-1859) et de mettre au jour les liens entre ces romans édifiants et les hantises exprimées par Baudelaire ou Edmond de Goncourt.

Mots-clés : édification, comtesse de Ségur, Victorine Monniot, Baudelaire, Goncourt, Chérie 


\section{Vanity for the use of young ladies:}

Good manners and good death in educational novels for Second Empire girls ABSTRACT: In the 1850es, the Second Empire settles in after five stormy decades that unveil the vanity of temporal power. The legitimist writers Sophie Rostoptchine, countess de Ségur, and Victorine Monniot, have left a deep imprint in the education of Second Empire young ladies. Their teaching, which taps on practical interpretation of Cahtolic catechism illustrate the Christian principles of renunciation taught by the memento mori while echoing the anguishes of the time. The article aims to study the presence of vanity as a pedagogical tool in Le Journal de Marguerite (1858) and the cycle of Sophie (1858-1859) and to highlight the links between these edifying novels and the fears expressed by Baudelaire and Edmond de Goncourt.

Keywords: edification, countess de Ségur, Victorine Monniot, Baudelaire, Goncourt, Chérie 